\title{
Using isothiocyanate excretion as a biological marker of Brassica vegetable consumption in epidemiological studies: evaluating the sources of variability
}

\author{
Jay H Fowke ${ }^{1, *}$, Jed W Fahey ${ }^{2}$, Katherine K Stephenson ${ }^{2}$ and James R Hebert ${ }^{1}$ \\ 'Department of Epidemiology and Biostatistics and the Nutrition Center, School of Public Health, University of South \\ Carolina and the South Carolina Cancer Center, Columbia, SC, USA: ${ }^{2}$ Department of Pharmacology and Molecular \\ Sciences, Brassica Chemoprotection Laboratory, Johns Hopkins University School of Medicine, Baltimore, MD, USA
}

\begin{abstract}
Objective: Brassica vegetable consumption (e.g. broccoli) leads to excretion of isothiocyanates (ITC) in urine. We evaluated the consistency of ITC as a biomarker for dietary Brassica vegetable consumption across the types of vegetables and methods of preparation used in Western societies, and across consumption levels. Design: A single-armed behavioural intervention with duplicate baseline assessment and post-intervention assessment. Urinary ITC excretion and estrogen metabolites were measured from 24-hour urine samples. Dietary intake was measured by a 24hour recall.

Setting: The behavioural intervention facilitated daily Brassica intake among participants by providing peer support, food preparation instruction, guided practice in a teaching kitchen, and other information.

Subjects: Thirty-four healthy free-living postmenopausal women who recently had a negative screening mammogram at the University of Massachusetts Medical Center. Results: Urinary ITC excretion and total Brassica intake followed the same pattern over the intervention. The ITC biomarker significantly predicted Brassica intake when Brassica consumption averaged about $100 \mathrm{~g} \mathrm{day}^{-1}$, but not when Brassica consumption averaged about $200 \mathrm{~g} \mathrm{day}^{-1}$. Urinary ITC levels were somewhat higher when more raw vegetables were consumed as compared to lightly cooked vegetables, while the types of Brassica consumed appeared to have only a small, non-significant effect on urinary ITC levels.

Conclusion: Urinary ITC excretion would be a good exposure biomarker among populations regularly consuming a vegetable serving/day, but may be less accurate among populations with greater intake levels or a wide range of cooking practices.
\end{abstract}

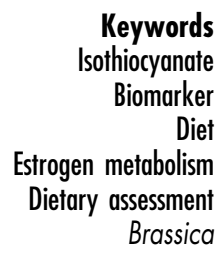

A diet rich in vegetables of the family Cruciferae, which in the USA consists primarily of Brassica vegetables (e.g. broccoli, green cabbage, Brussels sprouts), may reduce the risk of many common cancers ${ }^{1-8}$. Brassica vegetables are a well-known source of glucosinolates, $N$-hydroxysulfates with a variable aglycone group containing either an alkyl, alkenyl, thioalkyl, thioalkenyl, aryl, arylalkyl or indolyl moiety ${ }^{9,10}$. Glucosinolates are hydrolysed to their isothiocyanate congeners, or to nitriles, thiocyanates or other compounds by myrosinase, an enzyme in plant cells and in the human gut microflora ${ }^{11}$. These reaction products interact with various mammalian cellular and metabolic systems that are associated with cancer risk ${ }^{12-14}$, including Phase 2 detoxification enzymes (e.g. glutathione-S-transferase (GST)) that protect animals and their cells against oxidative stress, carcinogenesis and mutagenesis $^{15-19}$.

While there is an abundance of evidence illustrating a biological response to Brassica phytochemicals that is consistent with reduced cancer risk, there is only sparse and inconsistent prospective cohort or case-control epidemiologic evidence that Brassica consumption reduces cancer incidence or mortality. One explanation for this lack of epidemiological evidence might be that Brassica consumption is not adequately measured. Large case-control or cohort studies usually measure dietary 
intake with a food-frequency questionnaire (FFQ). These dietary instruments query only a limited number of foods, request average portion sizes, and rely on long-term memory to recall past dietary practices ${ }^{20}$. Additionally, there is a growing body of evidence to suggest that data from such self-reported dietary assessment techniques are influenced by participants' characteristics, including their psychological profiles ${ }^{21}$. The large potential public health benefit of even a small percentage reduction in cancer incidence suggests the need for a better method to estimate glucosinolate intake in free-living study populations.

Recently, an assay was developed to measure isothiocyanates and their metabolites in human urine ${ }^{11,22,23}$. Biological markers for dietary intake are not susceptible to reporting errors that limit self-report, especially FFQ $\mathrm{data}^{24}$, and therefore urinary ITC excretion might provide a less biased way of assessing Brassica intake in largescale studies. In several highly controlled metabolic studies, urinary isothiocyanate excretion levels (ITC) were consistent with the amount of Brassica administered to the study participant ${ }^{11,25-27}$. Seow and colleagues found that categories of total Brassica intake as measured by FFQ significantly followed the trend across categories of urinary ITC levels ${ }^{23}$. This Asian study population consumed moderate daily amounts of Brassica (40 $\mathrm{g} \mathrm{day}^{-1}$ ), primarily as Chinese cabbage, and cooking practices were not evaluated.

There are several sources of variability that could lead to substantial error in estimating Brassica intake by means of urinary ITC analysis. A further evaluation of these errors could benefit research studies intending to use ITC as a biomarker for dietary intake. The glucosinolate content of Brassica vegetables varies across species and cultivars, and depends on numerous environmental variables such as soil conditions under which the vegetables are grown ${ }^{9,15}$. Not all glucosinolates are equally likely to be converted to detectable isothiocyanates ${ }^{10,11}$. Therefore the quality of ITC as a biomarker for Brassica intake may depend upon the types of vegetables consumed. In addition, post-harvest handling, plant age, vegetable preparation and individual metabolic activity further affect glucosinolate concentration, effective dose and biological activity $9,15,23,28-31$.

An intensive 4-week dietary intervention was designed and implemented in order to evaluate the physiological response to increased Brassica vegetable consumption and to develop new functional-food assessment approaches in healthy free-living people. Since the intervention was of high intensity and relatively short duration, it provided an ideal opportunity to compare estimated Brassica intake from two independent sources: a well-regarded dietary assessment standard and the ITC biomarker of Brassica exposure. Over the three phases of the intervention, the same group of 34 participants consumed low, moderate and high amounts of Brassica, enabling us to evaluate the consistency between ITC excretion and self-reported Brassica consumption across different levels of consumption, and to evaluate the ability of ITC to track changes in dietary intake within individuals. Variability in ITC excretion with vegetable type and vegetable preparation are considered. We have previously reported that greater Brassica intake leads to a shift in the estrogen composition in these study participants, such that the amount of 2-hydroxyestrone increased relative to the amount of $16 \alpha$-hydroxyestrone ${ }^{32}$. We compared urinary ITC levels, as a marker for dietary Brassica intake, to urinary estrogen metabolite levels known to be affected by dietary Brassica intake.

\section{Materials and methods}

\section{Study participants}

Several aspects of this study have been described previously $^{32}$. Study participants were healthy, free-living, postmenopausal women who had received screening mammographic services within the past year. Participants were over 45 years of age and without a menstrual cycle during the previous 12 months. Tobacco-users or women who regularly consumed more than two alcoholic drinks per day were excluded. Additionally, subjects using any prescription or non-prescription hormones, diabetes medication, antibiotics, herbal remedies, or who were under a physician-recommended diet, were excluded. The average age of the 34 women participating in the study was 61.8 years ( $\mathrm{SD}=8.1$, range: $49-77)$. Twenty-five subjects were married and 17 had achieved at least a college degree. Fourteen women were employed, primarily in service-oriented positions such as nursing or in managerial/office jobs.

Study participants attended a series of four classes designed to facilitate the addition of Brassica vegetables to the daily diet. Class discussions were led by a registered dietitian, but relied on peer support and peer modelling to motivate adherence. Content focused on problem-solving skills and overcoming barriers associated with the dietary change. A strong emphasis was placed on vegetable preparation, and participants were encouraged to eat either raw or lightly steamed vegetables. Participants practised vegetable preparation skills through guided meal preparation in a teaching kitchen.

\section{Study design and data collection}

The study design and sample collection schedule are illustrated in Fig. 1. Study participants provided two 24hour urine samples prior to the intervention period, which are referred to as 'Baseline-1' and 'Baseline-2'. Additional 24-hour urine samples were collected during the last week of the intervention phase (referred to as 'Intervention') and two weeks after the conclusion of the intervention ('Postintervention'). Both written and oral 


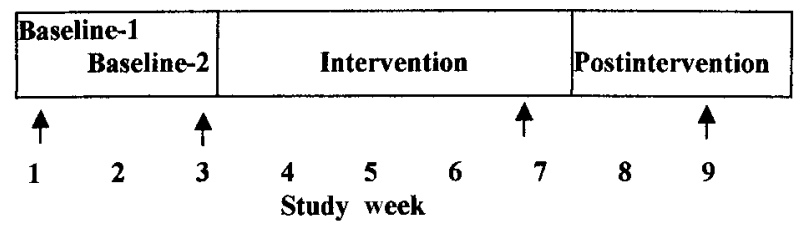

Fig. 1 Study design and data collection schedule: each arrow indicates a week during the study phase in which three 24-hour recalls were administered and a 24-hour urine sample was collected

instructions concerning 24-hour urine collection were administered to all participants. Subjects also were advised to avoid prepared mustard and horseradish during the two days prior to the urine collection, as these condiments are made from Cruciferous vegetables and have significant quantities of allyl isothiocyanate. Each opaque urine collection bottle contained $2.0 \mathrm{~g}$ ascorbic acid as a preservative. Urine samples were delivered to project staff within 1 day of collection, and most were delivered on the same day of collection. Upon arrival, total volumes of urine were recorded, and aliquots were stored at $-80^{\circ} \mathrm{C}$. Urine aliquots were shipped on dry ice to Baltimore for ITC analysis.

During each week in which urine was collected, three 24-hour diet recalls (24HR) were administered to each participant. Participants were telephoned on three randomly assigned days (two weekdays and one weekend day) and asked to describe their diet on the preceding day. A structured interview protocol was strictly followed. Highly trained registered dietitians conducted all interviews, and participants were provided a two-dimensional chart of typical foods to assist with portion size estimation. Nutrient calculations were performed using the Nutrition Data System software, developed by the Nutrition Coordinating Center, University of Minnesota, Minneapolis, MN (Food Database: 13A; Nutrient Database: 28$)^{33}$. There were no missing nutrient values in this analysis. Data from the $24 \mathrm{HR}$ within each week were averaged for each participant. Of the 408 calls assigned to the 34 participants, 401 calls were completed (98.3\%). From each 24HR log, the amount (grams) of Brassica vegetable reported were combined within each day by vegetable type and by cooked or raw status, and then averaged across all 24HR during the week. Brassica vegetables reported as cooked were adjusted to reflect raw grams consumed.

Questionnaires were administered throughout the study, first to collect demographic and breast cancerrelated data, then to monitor for changes in medication use, physical activity, occupational status, or alcohol use. The psychological constructs 'Social Approval' and 'Social Desirability', along with demographic and other data, were measured by questionnaire during the baseline study phase $\mathrm{s}^{34,35}$.

\section{Isothiocyanate laboratory analysis}

Samples $(1.5 \mathrm{ml})$ from each urine collection were thawed and centrifuged (200 $\mathrm{g}$ for $5 \mathrm{~min}$ at $4^{\circ} \mathrm{C}$ ) to remove particulate matter. The cyclocondensation reaction ${ }^{22}$ with urine was carried out in $4 \mathrm{ml}$, screw-topped glass vials in a final volume of $2.0 \mathrm{ml}$ that contained 200 or $500 \mu \mathrm{l}$ of urine and enough water to total $500 \mu \mathrm{l}, 0.5 \mathrm{ml}$ of $500 \mathrm{mM}$ sodium borate buffer ( $\mathrm{pH} 9.25$ ), and $1.0 \mathrm{ml}$ of $40 \mathrm{mM} \mathrm{1,2-}$ benzenedithiol in methanol. The vials were flushed with $\mathrm{N}_{2}$ gas, sealed with Teflon-lined septa, and the contents were mixed with a Vortex mixer and incubated for $2 \mathrm{~h}$ at $65^{\circ} \mathrm{C}$. Samples were then cooled to room temperature, briefly centrifuged ( $350 \mathrm{~g}$ for $5 \mathrm{~min})$, and loaded into a Waters WISP Autosampler ${ }^{\circledR}$. Aliquots $(200 \mu \mathrm{l})$ of each reaction mixture were injected on to a reverse-phase high-performance liquid chromatography (HPLC) column (Partisil $10 \mu \mathrm{m}$ ODS-2, $4.5 \times 250 \mathrm{~mm}$; Whatman, Clifton, $\mathrm{NJ})$ and eluted isocratically with $80 \%$ methanol $/ 20 \%$ water $(\mathrm{v} / \mathrm{v})$ at a flow rate of $2 \mathrm{ml} \mathrm{min}^{-1}$. The cyclocondensation product peak, 1,3-benzodithiole-2-thione, was eluted at c. $5.0 \mathrm{~min}$, and its area was integrated at $365 \mathrm{~nm}$ using a Waters Photodiode Array detector (Waters Millenium Software ${ }^{\circledR}$, Version 2.15.01).

Three sets of controls were included with each analytical run: (1) purified cyclocondensation product (200 $\mu \mathrm{l}$ of $2.5,5.0$ and $10.0 \mu \mathrm{M}$ solutions) was injected to assess the validity of the standard curve; (2) a reaction mixture containing only 1,2-benzenedithiol was included to ensure that no peak is given by 1,2-benzenedithiol alone; and (3) three concentrations (2.5, 5.0 and $10.0 \mu \mathrm{M}$ ) of the $N$-acetylcysteine derivative of allyl isothiocyanate were analysed with and without 1,2-benzenedithiol to ensure that the cyclocondensation reaction went to completion. Standard curves, assay reproducibility, linearity of response and storage characteristics of urine samples were all as detailed in Shapiro et al. ${ }^{11}$. Urinary ITC concentration $\left(\mu \mathrm{mol} \mathrm{ml}{ }^{-1}\right.$ ) was multiplied by the volume of urine collected during the 24-hour period ( $\mathrm{ml}$ ), to give $\mu$ mol daily ITC excretion.

\section{Urinary estrogen metabolites}

Urinary 2-hydroxyestrone (2HE) and 16 $\alpha$-hydroxyestrone (16HE) were measured using a solid-phase enzyme immunoassay kit from Immuna Care Corporation (Bethlehem, PA $)^{32,36}$. All assays were performed on samples in triplicate, in random order, within one batch, and by a single technician who was masked as to the sequence of the sample collection. The intra-assay coefficients of variation (CV) for $2 \mathrm{HE}$ and $16 \mathrm{HE}$ were each $4.0 \%$ and inter-assay CVs were $10.0 \%$ and $9.9 \%$, respectively. Standard urine samples were obtained from women of a similar age and estrogen level as the study participants.

\section{Statistical analysis}

A descriptive analysis of dietary Brassica intake and urinary ITC excretion is presented at all four measurement 
Table 1 Isothiocyanate (ITC) excretion or self-reported Brassica intake

\begin{tabular}{|c|c|c|c|c|c|c|c|c|c|c|}
\hline \multirow[b]{2}{*}{ Study phase } & \multicolumn{5}{|c|}{ ITC $(\mu \mathrm{mol} / 24 \mathrm{~h})^{\star}$} & \multicolumn{5}{|c|}{ Brassica $(\mathrm{g} / 24 \mathrm{~h}) \dagger$} \\
\hline & Min & Q1 & Median & Q3 & Max & Min & Q1 & Median & Q3 & Max \\
\hline Baseline-1 & $0(5)$ & 0.58 & 1.96 & 5.21 & 11.34 & $0(15)$ & 0 & 3.9 & 20.3 & 52.6 \\
\hline Baseline-2 & $0(12)$ & 0 & 0.51 & 4.12 & 38.97 & $0(20)$ & 0 & 0 & 10.4 & 75 \\
\hline Intervention & 0.92 & 9.46 & 16.90 & 37.69 & 145.83 & 53.6 & 149.8 & 180.9 & 245.4 & 371.5 \\
\hline Postintervention & $0(1)$ & 5.79 & 12.49 & 25.98 & 84.09 & $0(3)$ & 50.7 & 105.0 & 166.3 & 241.8 \\
\hline
\end{tabular}

Min: minimum value; Q1: 25th percentile; Q3: 75th percentile; Max: maximum value.

( ): Number of participants with no detectable ITC or reporting $0 \mathrm{~g}$ Brassica intake.

* ITC excretion measured in 24-hour urine samples collected during each phase of the study.

† Brassica consumption measured with three 24-hour recalls $(24 \mathrm{HR})$ during the same week that a 24-hour urine sample was collected.

times within the intervention (i.e. Baseline-1, Baseline-2, Intervention and Postintervention). The analysis focused on the association between Brassica intake and ITC excretion during the Intervention and Postintervention study phases because few participants consumed Brassica at Baseline, and there was insufficient variability in the dietary and urinary data to perform the detailed analysis within this study phase. The isothiocyanate data were natural $\log (\ln )$ transformed to better meet assumptions of the statistical analysis. Brassica vegetable intake data during the Intervention and Postintervention study phases approximated a normal distribution. Pearson correlation coefficients and linear regression coefficients were used to assess the cross-sectional associations. Regression coefficients reflect the amount of urinary ITC excretion $\left(\ln \left(\mu \mathrm{mol} \mathrm{day}{ }^{-1}\right)\right)$ due to each unit change (e.g. $\mathrm{g} \mathrm{day}^{-1}$ ) in the dietary parameter. Vegetable-specific associations with urinary ITC excretion were identified using partial correlation coefficients, or by simultaneously including each vegetable type in a linear regression model that predicts ITC excretion. Similarly, the amounts of Brassica intake consumed as cooked or raw were simultaneously included in a linear regression model.

We evaluated the ability of urinary ITC levels to track individual changes in Brassica intake or to induce a change in estrogen metabolism. Measurements across the two baseline time points were not significantly different, and each subject's two baseline values were averaged together for calculation of the change scores, in order to provide the most stable baseline estimate. Change scores for Brassica, ITC levels or the relative amounts of estrogen metabolites (2HE/16HE ratio) were computed
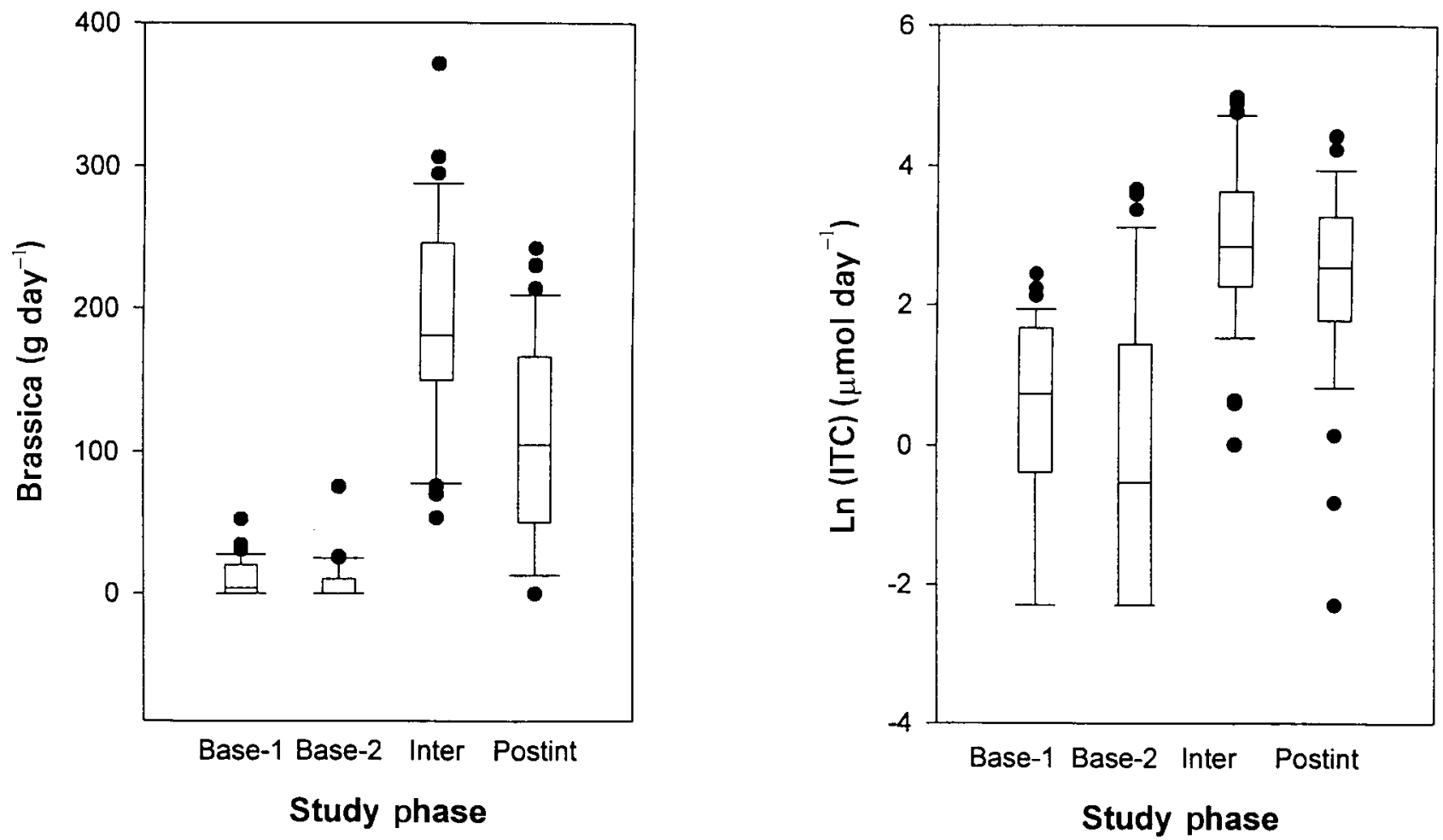

Fig. 2 The distribution of Brassica vegetable consumption and urinary isothiocyanate (ITC) excretion across phases of the study (Base-1, Baseline-1; Base-2, Baseline-2; Inter, Intervention; Postint, Postintervention). Horizontal lines at 5th, 25th, 50th, 75th and 95th percentiles 
by subtraction of the Baseline value from either the Intervention or the Postintervention value. Paired $t$-tests were used to compare changes in diet or urinary measures between any two time points. Correlation coefficients and regression coefficients were adjusted for baseline (log-transformed) ITC values, in order to reduce the influence of an extreme baseline value on the change score (i.e. regression to the mean).

\section{Results}

Reported Brassica vegetable consumption and urinary ITC excretion are summarised in Table 1 and Fig. 2. At Baseline, participants' Brassica intake was similar across the two baseline measures $(P=0.37)$, and averaged about $9 \mathrm{~g}$ of vegetable per day. Brassica consumption increased during the intervention to $193 \mathrm{~g} \mathrm{day}^{-1}(P<0.001$, Average Baseline value vs. Intervention), and all participants reported greater Brassica consumption during the intervention. At Postintervention, average Brassica consumption decreased by $84 \mathrm{~g} \mathrm{day}^{-1}(P<0.001$ for Intervention vs. Postintervention). Broccoli, cabbage and Brussels sprouts were most commonly consumed (Intervention: 50.5, 42.5 and $75.7 \mathrm{~g} \mathrm{day}^{-1}$; Postintervention: 27.0, 14.8 and $43.1 \mathrm{~g} \mathrm{day}^{-1}$, respectively).

Urinary ITC levels were lowest at the two Baseline time points, and these Baseline ITC levels were not significantly different $(P=0.24)$. ITC was non-detectable in 17 urine samples at Baseline, whereas all urine samples obtained during the Intervention phase of the study contained detectable ITC. Group-average ITC excretion levels followed the trend of Brassica intake, with significant increases from Baseline to Intervention $(P<$ $0.01)$, and a decrease from the Intervention to Postintervention phase of the study $(P<0.01)$.

The association between individual-level ITC excretion and reported Brassica intake was evaluated within each measurement period of the study (Table 2, Fig. 3). The scatter plots of Fig. 3 illustrate the unstable association between Brassica intake and ITC excretion during the two Baseline collection periods, when Brassica consumption was very low and sporadic. Any linear association was due to a few highly influential values, and therefore we restrict further analyses to the Intervention and Postintervention study phases. During the Intervention period, where intake was highest, there was only a weak and non-significant association between urinary ITC level and total Brassica intake or vegetable specific intake. During the Postintervention, where intake was moderate, there was a significant association between ITC excretion and Brassica intake $(r=0.58, P<0.01)$, at which time each $\mathrm{g} \mathrm{day}^{-1}$ of Brassica led to an increase of $0.24 \mathrm{log}$ units $\left(\log \left(\mu \mathrm{mol} \mathrm{day}{ }^{-1}\right)\right)$ in urinary ITC levels. Adjustment for macronutrient intake (protein $\mathrm{g} \mathrm{day}^{-1}$, fat $\mathrm{g} \mathrm{day}^{-1}$, energy kcal day ${ }^{-1}$, carbohydrate $\mathrm{g} \mathrm{day}^{-1}$ ) did not affect the association between Brassica intake and ITC excretion.

The commonly consumed vegetables (i.e. broccoli, cabbage and Brussels sprouts) appeared to contribute proportionally equivalent amounts of ITC to the content of urine at Postintervention, but there was greater disparity during the Intervention. Brussels sprouts intake was highest during the Intervention, and certain glucosinolates common in Brussels sprouts might be less likely to contribute to ITC in urine. When Brussels sprouts consumption was removed from the total amount of Brassica consumed, the associations improved slightly during the Intervention $(r=0.21, P=0.23 ; b=0.06$, $95 \%$ CI $(0.04,0.17))$, but regression coefficients at Postintervention decreased slightly $(r=0.46, P=0.006$; $b=0.23,95 \%$ CI $(0.07,0.39))$. Raw vegetable intake tended to be more strongly associated with ITC levels as compared with cooked vegetable intake.

Dietary interventions and metabolic (in-patient) studies often analyse changes in a biochemical measure as an individual-level marker of exposure change. As described

Table 2 Association between isothiocyanate excretion and total Brassica intake, and by vegetable type or vegetable preparation

\begin{tabular}{|c|c|c|c|c|c|c|c|c|}
\hline \multirow[b]{2}{*}{ Brassica } & \multicolumn{4}{|c|}{ Intervention } & \multicolumn{4}{|c|}{ Postintervention } \\
\hline & $r$ & $P$ & $b$ & $95 \% \mathrm{Cl}$ & $r$ & $P$ & $b$ & $95 \% \mathrm{Cl}$ \\
\hline \multicolumn{9}{|l|}{ Model $1^{*}$} \\
\hline Total & 0.14 & 0.45 & 0.04 & $-0.06,0.15$ & 0.58 & $<0.01$ & 0.24 & $0.12,0.36$ \\
\hline \multicolumn{9}{|l|}{ Model $2 \dagger$} \\
\hline Broccoli & 0.12 & 0.51 & 0.06 & $-0.12,0.25$ & 0.51 & $<0.01$ & 0.38 & $0.14,0.63$ \\
\hline Cabbage & -0.05 & 0.76 & -0.03 & $-0.23,0.17$ & 0.32 & 0.08 & 0.31 & $-0.03,0.66$ \\
\hline Brussels sprouts & -0.05 & 0.78 & -0.02 & $-0.18,0.14$ & 0.50 & $<0.01$ & 0.34 & $0.11,0.57$ \\
\hline Other & 0.32 & 0.08 & 0.23 & $-0.02,0.49$ & 0.08 & 0.67 & 0.05 & $-0.20,0.31$ \\
\hline \multicolumn{9}{|l|}{ Model 3ł } \\
\hline Cooked & 0.07 & 0.68 & 0.02 & $-0.09,0.14$ & 0.47 & $<0.01$ & 0.20 & $0.06,0.34$ \\
\hline Raw & 0.23 & 0.18 & 0.12 & $-0.06,0.31$ & 0.44 & 0.01 & 0.40 & $0.10,0.70$ \\
\hline
\end{tabular}

Isothiocyanate (ITC) measured from 24-hour urine samples collected during each phase of the study. Brassica consumption measured by three 24-hour recalls during each week in which a urine sample was collected for isothiocyanate measurement.

${ }^{*}$ Model 1: $\log \left(\right.$ ITC)$=$ Total Brassica $\left(20 \mathrm{~g} \mathrm{day}^{-1}\right)$.

† Model 2: $\log ($ ITC $)=$ Broccoli $\left(20 \mathrm{~g} \mathrm{day}^{-1}\right)+$ Cabbage $\left(20 \mathrm{~g} \mathrm{day}^{-1}\right)+$ Brussels sprouts $\left(20 \mathrm{~g} \mathrm{day}^{-1}\right)+\mathrm{Other}\left(20 \mathrm{~g} \mathrm{day}^{-1}\right)$.

† Model 3: $\log ($ ITC $)=$ Cooked Brassica $\left(20 \mathrm{~g}_{\text {day }}{ }^{-1}\right)+$ Raw Brassica $\left(20 \mathrm{~g} \mathrm{day}^{-1}\right)$.

Partial Pearson correlation coefficients adjusted for other variables in model. 


\section{Baseline-1}

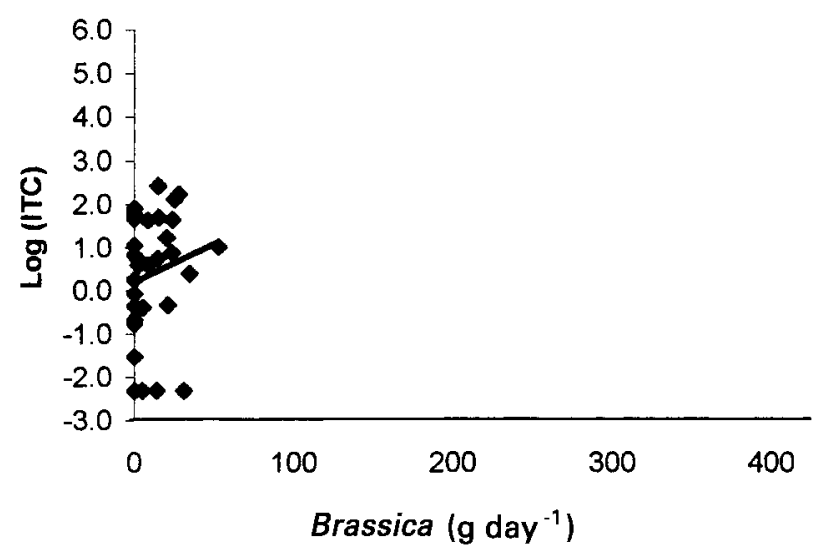

Intervention

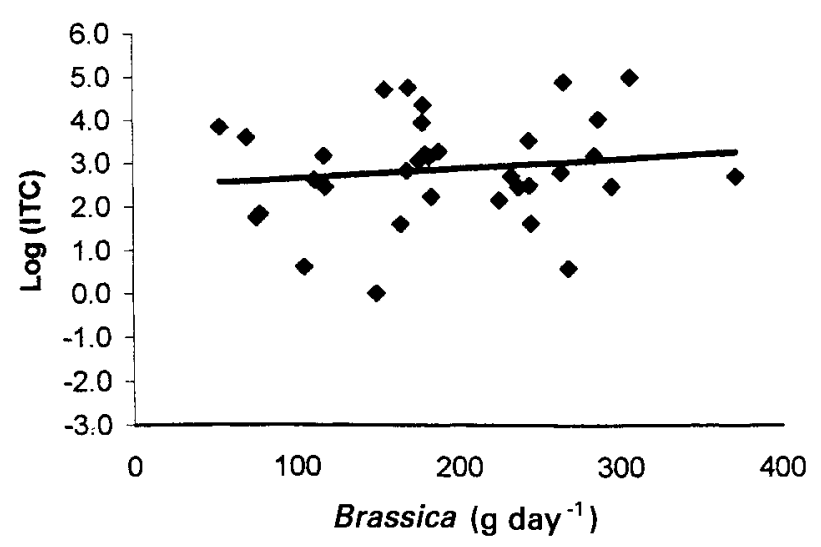

Baseline-2

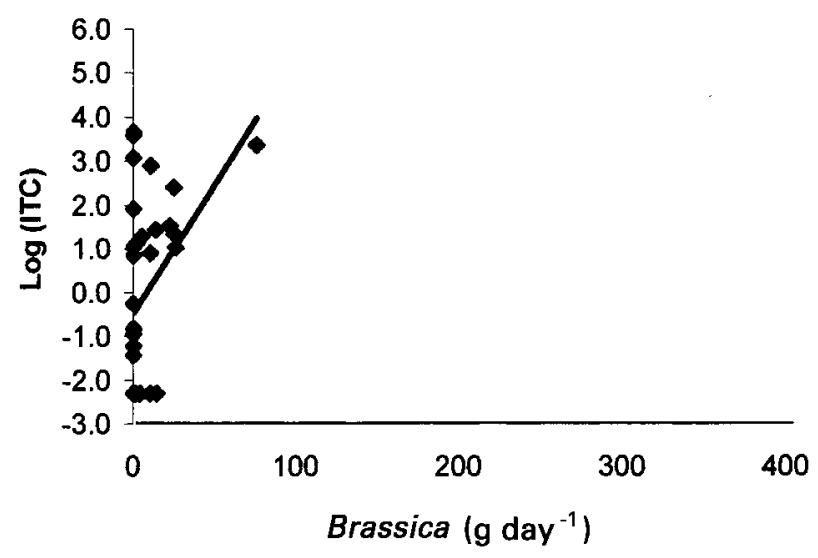

Postintervention

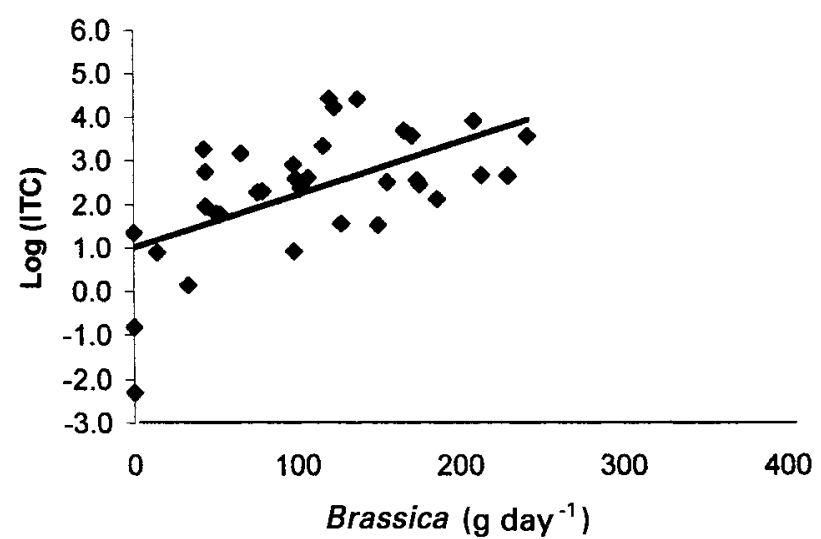

Fig. 3 Brassica intake and isothiocyanate excretion within the same participants consuming different levels of Brassica

in the Methods section, changes in Brassica or urinary ITC levels were calculated by subtracting the average Baseline values from values at either the Intervention or Postervention study phase. Moderate change in Brassica intake (Postintervention-Baseline) was associated with a significant change in urinary ITC levels, where each $20 \mathrm{~g} \mathrm{day}^{-1}$ increase in Brassica intake led to a $0.24 \log$-unit increase in urinary ITC excretion (Table 3). In contrast, larger change in Brassica intake
(Intervention-Baseline) was associated with almost no change in ITC levels.

In order to explore the consistency between urinary ITC levels and a physiological response to Brassica vegetable intake, the urinary estrogen metabolite ratio 2-hydroxyestrone: $16 \alpha$-hydroxyestrone (2HE/16HE) was regressed on urinary ITC excretion levels. Greater urinary ITC levels were not significantly associated with a higher urinary $2 \mathrm{HE} / 16 \mathrm{HE}$ ratio within either the Intervention or

Table 3 Association between change in grams of Brassica vegetable intake per day, and change in isothiocyanate (ITC) excretion

\begin{tabular}{|c|c|c|c|c|c|c|c|c|}
\hline \multirow[b]{2}{*}{ Brassica } & \multicolumn{4}{|c|}{ Intervention-Baseline } & \multicolumn{4}{|c|}{ Postintervention-Baseline } \\
\hline & $r$ & $P$ & $b$ & $95 \% \mathrm{Cl}$ & $r$ & $P$ & $b$ & $95 \% \mathrm{Cl}$ \\
\hline Total $\left(20 \mathrm{~g} \mathrm{day}^{-1}\right)$ & 0.18 & 0.31 & 0.06 & $-0.05,0.17$ & 0.57 & $<0.01$ & 0.24 & $0.11,0.36$ \\
\hline
\end{tabular}

Ln (Isothiocyanate (ITC)) measured from 24-hour urine samples collected during each phase of the study. Brassica consumption measured by three 24-hour recalls during each week in which a urine sample was collected for isothiocyanate measurement.

Change scores calculated by subtracting the average of the two Baseline measurements for dietary intake or log(ITC) levels from values at Intervention or Postintervention.

Model: $\Delta \log \left(\right.$ ITC) $=\Delta$ Brassica+log $\left(\right.$ ITC $\left._{\text {Baseline }}\right)$. 
Table 4 Association between urinary isothiocyanate excretion level and the $2 \mathrm{HE} / 16 \mathrm{HE}$ estrogen metabolite ratio

\begin{tabular}{|c|c|c|c|c|}
\hline & $r$ & $P$ & $b$ & $95 \% \mathrm{Cl}$ \\
\hline \multicolumn{5}{|l|}{ Cross-sectional $^{\star}$} \\
\hline Intervention & 0.13 & 0.45 & 0.12 & $-0.21, \quad 0.45$ \\
\hline $\begin{array}{l}\text { Postintervention } \\
\text { Change† }\end{array}$ & -0.04 & 0.83 & -0.05 & $-0.51, \quad 0.41$ \\
\hline Intervention-Baseline & -0.18 & 0.29 & -0.20 & $-0.61, \quad 0.18$ \\
\hline Postintervention-Baseline & -0.49 & $<0.01$ & -0.74 & $-1.21,-0.26$ \\
\hline
\end{tabular}

* Cross-sectional model: $2 / 16=\log ($ ITC).

† Change model: $\Delta 2 / 16=\Delta(\log I T C)+$ ITC $_{\text {Baseline }}$

Postintervention study phase (Table 4). Adjustment for the previously described dietary factors led to a stronger association during the Intervention $(b=0.28,95 \%$ $\mathrm{CI}(-0.14,0.69))$, but had no impact on the Postintervention association $(b=-0.04,95 \%$ CI $(-0.34,0.26))$. Unexpectedly, increased ITC excretion from Baseline to Postintervention was significantly associated with lower urinary 2HE/16HE levels $(r=-0.49, \quad P<0.01)$. No outliers or highly influential data points were evident. Statistical adjustment for changes in macronutrient intake during these time intervals did not change the interpreted results (Intervention-Baseline: $b=-0.24, \quad 95 \%$ CI $(-0.53,0.03) ; \quad$ Postintervention-Baseline: $b=-0.41$, 95\% CI $(-0.62,-0.19))$.

\section{Discussion}

Nutritional epidemiology often evaluates the association between the macro- or micronutrient components of the diet and disease risk. There is growing evidence that the non-nutrient components of the diet could impact cancer risk. Examples include genestein derived from soy-foods, enterolactone from grain-foods, and isothiocyanates from Brassica vegetables. However, it is difficult to measure exposure to these food components accurately. Typical FFQs do not query an exhaustive listing of these vegetables or factors that may modify the phytochemical content. Urinary markers of dietary intake are less susceptible to reporting bias and might provide a chemical-specific exposure level. We created a model dietary intervention developed in part to design and evaluate dietary assessment strategies for functional food intake. In this intervention, the same participants consumed very high or moderate levels of Brassica vegetables, providing the unique opportunity to evaluate the performance of the biomarker across a range of intakes within the same individuals. The $24 \mathrm{HR}$ is a dietary assessment method that measures the current diet without resorting to standardised comparison portions in order to estimate the quantity of food consumed. This method provides the least biased approach to estimating dietary intake within an intervention ${ }^{37,38}$.

We found that ITC excretion was a better predictor of Brassica intake when the group consumed a moderate level of Brassica. Previously, Seow and colleagues found a greater discrepancy between estimated Brassica intake and ITC excretion among those participants who consumed more Brassica, and by GSTT1 gene expression ${ }^{23}$. The metabolism of ITC may vary across individuals according to the expression and activity of GST and other metabolic enzymes, such that the urinary biomarker no longer represents dietary intake beyond a certain level of intake. In this study of US women, that threshold appears to be between $100 \mathrm{~g} \mathrm{day}^{-1}$ and $200 \mathrm{~g} \mathrm{day}^{-1}$, on average. There was a dose-response pattern during the Postintervention study phase, when intake was moderate. The linear trend was lost with higher average intake. Additionally, there was a marginally significant association between changes in ITC excretion levels and changes in Brassica intake among free-living participants at Postintervention.

The amount of Brassica consumed at Postintervention is a better representation of the amounts consumed in many Asian regions. For example, Seow and colleagues found that Cruciferous intake averaged about $40 \mathrm{~g} \mathrm{day}^{-1}$ in Singapore ${ }^{23}$. Variance measures were not provided, but there was likely a wide distribution reaching into the ranges observed during the Postintervention phases of this study. According to food-disappearance data, the Japanese consume far more cabbage (19 $\left.\mathrm{g} \mathrm{day}^{-1}\right)$, Chinese cabbage (22 $\mathrm{g} \mathrm{day}^{-1}$ ) and other Brassica vegetables which are rarely consumed in Western cultures ${ }^{39}$. This study indicates that populations which routinely consume Brassica do not appear to do so to such an extent that the urinary ITC marker would be unreliable. Consumption of Brassica vegetables in the United States has been estimated at $11 \mathrm{~g} \mathrm{day}^{-1}$ in an analysis of food production data ${ }^{30}$; or about 2 servings per week in a large dietary survey ${ }^{40}$. These intake levels are similar to our Baseline measures, indicating that urinary ITC levels may be an unreliable estimate of Brassica intake in North American study populations.

Different Brassica vegetables have different glucosinolate concentrations, and the spectrum of glucosinolates differs from vegetable to vegetable. Although Brussels sprouts are a rich source of glucosinolates, the predominant glucosinolates include progoitrin, a $\beta$-hydroxyalkenyl glucosinolate that is hydrolysed by the enzyme myrosinase to produce nitriles, epithionitriles and oxazolidine-2-thiones, and not isothiocyanates. Progoitrin metabolites do not react to produce ITC in the human body, and these progoitrin metabolites do not react in the cyclocondensation reaction $^{22}$. Brussel sprouts consumption was very high during the Intervention phase. However, these results might suggest only the slightest variation in ITC excretion due to variation in the patterns of consumed Brassica.

As a generalisation, the United States' population typically consumes Brassica vegetables after cooking. Glucosinolates are water-soluble and leach into the 
cooking water with vegetable boiling, decreasing the

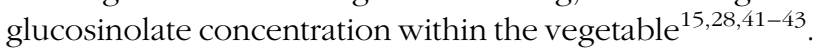
When thoroughly cooked Brassica vegetables are administered to subjects, plant myrosinase is inactivated, and essentially all of the glucosinolates/isothiocyanates are presented to the subject's digestive tract in glucosinolate form $^{11}$, and additional metabolic/enzymatic steps are required to release the ITC component from the glucosinolate. Alternatively, it could be possible that very light cooking releases myrosinase without enzyme inactivation, leading to increased glucosinolate metabolism. Requiring that participants consume only raw Brassica would so highly control the study that results would not be applicable to free-living women, and such a restriction would likely decrease compliance to the intervention. Study participants were instructed during the intervention classes to prepare Brassica vegetables using techniques that prevent glucosinolates from leaching or degrading in the vegetable, with the goal of maintaining the glucosinolate content of the vegetables at the highest possible level. We found that both raw and cooked Brassica consumption contributed to urinary ITC levels, with raw consumption appearing to contribute a little more ITC to urine, suggesting that ITC excretion might be sensitive to the simplest of food preparation methods.

To further explore the use of ITC, we compared urinary ITC excretion levels to the $2 \mathrm{HE} / 16 \mathrm{HE}$ estrogen metabolite ratio. The indole glucosinolates derived from Brassica vegetables are converted in the body to aryl hydrocarbon receptor agonists ${ }^{44}$, and the activated receptor is able to induce the specific enzyme responsible for hydroxylation of estrone on the second carbon (CYP1A1, CYP1A2), producing 2-hydroxyestrone rather than the highly estrogenic and genotoxic $16 \alpha$-hydroxyestrone. The ratio of these metabolites, $2 \mathrm{HE} / 16 \mathrm{HE}$, is currently under evaluation as an endocrine biomarker for breast cancer risk $^{45-50}$. We have found that Brassica consumption increases the $2 \mathrm{HE} / 16 \mathrm{HE}$ ratio in this study population, consistent with a reduced risk of breast cancer ${ }^{32}$. In contrast, urinary ITC levels, derived from Brassica vegetables, were not associated with the $2 \mathrm{HE} / 16 \mathrm{HE}$ ratio. The common non-isothiocyanate metabolites (e.g. nitriles, thiocarbamates, epithionitriles, oxazolidine-2thiones and various indole derivatives such as indole-3carbinol and indole-3-acetonitrile) are not detected by this assay. Therefore, the urinary ITC index may be less informative as a biomarker for Brassica when the hypothesised disease mechanism involves indole glucosinolates.

To our knowledge, this is the first attempt to adjust for other dietary constituents. Nutrient intake is able to affect drug metabolism ${ }^{51}$ and phytoestrogen excretion, either through induction or inhibition of Phase 1 or Phase 2 enzymes responsible for metabolism, or through increasing or decreasing the likelihood of faecal excretion over urinary excretion of the agent. Log-transformed urinary ITC excretion levels were not associated with dietary macronutrient intake, and adjustment for these nutrients did not affect the associations between ITC levels and either Brassica intake or urinary $2 \mathrm{HE} / 16 \mathrm{HE}$ values. We did not explore the effects of fibre intake on ITC excretion because increased Brassica consumption leads directly to both greater fibre intake and ITC excretion, limiting our ability to identify this independent association in this study.

It is possible that these motivated participants misreported their diet; however, there is little evidence to suggest that these study results are due to dietary misreport. It is conceivable that participants over-reported Brassica intake in order to achieve the appearance of compliance. The psychological scales 'social desirability' and 'social approval' have been previously evaluated for their effect on dietary self-report ${ }^{21}$. Social desirability describes a defensive mechanism where one is likely to present oneself in a fashion consistent with social norms and to avoid criticism, while social approval scores are associated with an intrinsic need to seek a positive response to a testing situation ${ }^{21,34,35}$. Consistent with the theoretical construct of the scales, the social approval scale was associated with Brassica intake during the Intervention phase $(r=0.33, P=0.05)$. Study personnel motivated participants to consume Brassica and provided feedback and problem-solving techniques, thus potentially creating a testing situation. During the Postintervention phase there was no contact with study personnel, thus reducing the 'pressure' to attain a specific level of Brassica intake. However, it is difficult to determine if participants with higher social approval scores met the testing challenge by consuming more Brassica or by reporting more Brassica. Social approval scores were not significantly correlated with ITC excretion $(r=0.06, P=$ $0.74)$, providing some support to the hypothesis that greater social approval scores were associated with misreport. However, adjustment with social approval scores had no effect on the regression coefficients during either the Intervention or Postintervention study phase. While there is some question as to the relationship between Brassica intake and social approval scores, misreport - if any - was small, and adjustment for social approval in the analysis did not alter the fundamental interpretation of the results.

It is unlikely that the differences in results over time are due to a training effect among participants. The duplicated baseline measurements were included to provide time for training. We have observed among women receiving repeated $24 \mathrm{HR}$ that all training occurs during the first $24 \mathrm{HR}$, and that the effect is very small relative to the overall variability in their diet. The 24HRs administered during the Intervention phase were the seventh, eighth and ninth calls received by these participants.

Other sources of error in the correspondence between 
urinary ITC levels and dietary Brassica consumption might be possible. Urinary ITC levels peak within several hours after consuming Brassica, but require one to three days to be completely excreted ${ }^{11}$. The $24 \mathrm{HR}$ assessment protocol contacted participants on three random days within the week of urine collection, and was designed to capture a representative sample of the habitual/regular Brassica intake during the week for that participant. It is possible that Brassica consumption during a day not queried by $24 \mathrm{HR}$ might have contributed to urinary ITC levels. This error may be greatest when Brassica consumption is relatively rare and sporadic, such as at Baseline, and less important with consistent and daily (or near daily) Brassica consumption (Intervention and Postintervention). None of the participants used tobacco, but it may be possible that environmental tobacco smoke contributed to urinary ITC levels ${ }^{11}$. Participants were instructed not to eat mustard or horseradish during the urine collection week, but eating foods prepared by other people may lead to condiment consumption without participant knowledge.

Improper urine sample handling or refrigeration by study participants may have led to microbial contamination, thus resulting in degradation of glucosinolate/ isothiocyanate in the urine and decreasing the association between ITC and self-reported Brassica intake. However, there was no difference in the time between reported completion of the urine collection and urine storage across the different phases of the study, making it unlikely that urine sample handling alone could explain the variable associations across the study. The vast majority of urine samples were returned to the research institution within hours of the completed urine collection. Further work is planned to identify a simple and effective ITCcompatible preservation protocol for epidemiological research.

In summary, categories of Brassica intake follow the pattern of categories of urinary ITC excretion, and there was a significant correlation between these two measures among participants who consumed an average of about $100 \mathrm{~g} \mathrm{day}^{-1}$. The cyclocondensation reaction is important because it provides an overall estimate of glucosinolate exposure across Brassica species and food preparation that standard FFQs do not capture. In using ITC as an exposure marker in large epidemiological studies, careful consideration should be given to variability in cooking practices, the amounts consumed, and the theorised biological mechanisms of the disease of interest.

\section{Acknowledgements}

The dietary intervention was conducted at the Division of Preventive and Behavioral Medicine, University of Massachusetts Medical School. Work in the authors' (JWF, KKS) laboratories was supported by generous gifts from Lewis and Dorothy Cullman and by a Program
Project grant (PO1CA 44530) from the National Cancer Institute, Department of Health and Human Services, Bethesda, MD, USA. We would like to thank Dr Theresa A Shapiro and Dr Christopher Longcope for thoughtful and insightful comments on an early draft of this manuscript.

\section{References}

1 Beecher CW. Cancer preventive properties of varieties of Brassica oleracea: a review. Am. J. Clin. Nutr. 1994; 59: 1166S-70S.

2 Block G, Patterson B, Subar A. Fruit, vegetables, and cancer prevention: a review of the epidemiological evidence. Nutr. Cancer 1992; 18: 1-29.

3 Steinmetz KA, Potter JD. Vegetables, fruit, and cancer prevention: a review. J. Am. Diet. Assoc. 1996; 96: 1027-39.

4 Michaud DS, Spiegelman D, Clinton SK, Rimm EB, Willett WC, Giovannucci EL. Fruit and vegetable intake and incidence of bladder cancer in a male prospective cohort. J. Natl. Cancer Inst. 1999; 91(7): 605-13.

5 Verhoeven DT, Goldbohm RA, van Poppel G, Verhagen H, van den Brandt PA. Epidemiological studies of Brassica vegetables and cancer risk. Cancer Epidemiol. Biomark. Prev. 1996; 5: 733-48.

6 Kohlmeier L, Su L. Cruciferous vegetable consumption and colorectal cancer risk: meta-analysis of the epidemiological evidence. FASEB J. 1997; 11(3): 2141.

7 Graham S, Dayai H, Swanson M, Mittleman A, Wilkinson G. Diet in the epidemiology of cancer of the colon and rectum. J. Natl. Cancer Inst. 1978; 61(3): 709-14.

8 Zhang S, Hunter D, Forman MR, Rosner BA, Speizer FE, Colditz GA, et al. Dietary carotenoids and vitamin A, C, and E and risk of breast cancer. J. Natl. Cancer Inst. 1999; 91(6): 547-56.

9 Rosa EAS, Heany RK, Fenwick GR, Portas CAM. Glucosinolates in crop plants. Horticult. Rev. 1997; 19: 99-215.

10 Fahey JW, Zalcmann AT, Talalay P. The chemical diversity and distribution of glucosinolates among plants. Phytochemistry 2001; 56: 5-51.

11 Shapiro TA, Fahey JW, Wade KL, Stephenson KK, Talalay P. Human metabolism and excretion of cancer chemoprotective glucosinolates and isothiocyanates of Cruciferous vegetables. Cancer Epidemiol. Biomark. Prev. 1998; 7: 1091-1100.

12 Cover CM, Hseih SJ, Tran SH, Hallden G, Kim GS, Bjeldanes $\mathrm{LF}$, et al. Indole-3-carbinol inhibits the expression of cyclindependent kinase- 6 and induces a G1 cell cycle arrest of human breast cancer cells independent of estrogen receptor signaling. J. Biol. Chem. 1998; 273(7): 3838-47.

13 Vang O, Frandsen H, Hansen KT, Nielsen JB, Andersen O. Modulation of drug-metabolising enzyme expression by condensation products of indole-3-ylcarbinol, and inducer in cruciferous vegetables. Pharmacol. Toxicol. 1999; 84: 5965.

14 Fares FA, Ge X, Yanni S, Rennert G. Dietary indole derivatives induce apoptosis in human breast cancer cells. Adv. Exp. Med. Biol. 1998; 451: 153-7.

15 Fahey JW, Stephenson KK. Cancer chemoprotective effects of cruciferous vegetables. Horticult. Sci. 1999; 34(7): 115963.

16 Prestera T, Talalay P. Electrophile and antioxidant regulation of enzymes that detoxify carcinogens. Proc. Natl. Acad. Sci. USA 1995; 92: 8965-9.

17 Talalay P. The war against cancer: new hope. Proc. Am. Phil. Soc. 1999; 143(1): 52-72.

18 Kensler TW. Chemoprevention by inducers of carcinogen 
detoxication enzymes. Environ. Health Persp. 1997; 105(Suppl.): 964-70.

19 Fahey JW, Talalay P. Antioxidant functions of sulforaphane: a potent inducer of phase 2 detoxication enzymes. Food Chem. Tox. 1999; 37(9): 973-9.

20 Willett W. Nutritional Epidemiology. New York: Oxford University Press, 1990.

21 Hebert JR, Clemow L, Pbert L, Ockene IS, Ockene JK. Social desirability bias in dietary self-report may compromise the validity of dietary intake measures. Int. J. Epidemiol. 1995; 24(2): 389-98.

22 Zhang Y, Wade KL, Prestera T, Talalay P. Quantitative determination of isothiocyanates, dithiocarbamates, carbon disulfide, and related thiocarbonyl compounds by cyclocondensation of 1,2-benzenedithiol. Anal. Biochem. 1996; 239: $160-7$.

23 Seow A, Shi C-Y, Chung F-L, Jiao D, Hankin JH, Lee H-P, et al. Urinary total isothiocyanate (ITC) in a population-based sample of middle-aged and older Chinese in Singapore: relationship with dietary total ITC and glutathione $S$ transferase M1/T1/P1 genotypes. Cancer Epidemiol. Biomark. Prev. 1998; 7: 775-81.

24 Kaaks R, Riboli E, Sinha R. Biochemical markers of dietary intake. In: In: Toniolo P, Boffetta P, Shuker D, Rothman N, Hulka B, Pearce N, eds. Application of Biomarkers in Cancer Epidemiology. Lyon: IARC, 1997; 103-26.

25 Getahun SM, Chung F-L. Conversion of glucosinolates to isothiocyanates in humans after ingestion of cooked watercress. Cancer Epidemiol. Biomark. Prev. 1999; 8: 447-51.

26 Chung F-L, Jiao D, Getahun SM, Yu MC. A urinary biomarker for uptake of dietary isothiocyanates in humans. Cancer Epidemiol. Biomark. Prev. 1998; 7: 103-8.

27 Chung F-L, Morse MA, Eklind KI, Lewis J. Quantitation of human uptake of the anticarcinogen phenethyl isothiocyanate after a watercress meal. Cancer Epidemiol. Biomark. Prev. 1992; 1: 383-8.

28 Goodrich RM, Anderson JL, Stoewsand GS. Glucosinolate changes in blanched broccoli and Brussels sprouts. J. Food Process. 1989; 13: 275-80.

29 Kushad MM, Brown AF, Kurlich AC, Juvik JA, Klein BP, Wallig MA, et al. Variation of glucosinolates in vegetable crops of the Brassica oleracea. J. Agric. Food Chem. 1999; 47(4): 1541-8.

30 Nugon-Baudon L, Rabot S. Glucosionolates and glucosinolate derivatives: implications for protection against chemical carcinogenesis. Nutr. Res. Rev. 1994; 7: 205-31.

31 London SJ, Yuan J-M, Chung FL, Gao Y-T, Coetzee BA, Ross RK, et al. Isothiocyanates, glutathione $S$-transferase M1 and T1 polymorphisms, and lung cancer risk: a prospective study of men in Shanghai, China. Lancet 2000; 356: 724-9.

32 Fowke JH, Longcope C, Hebert JR. Brassica vegetable consumption shifts estrogen metabolism in healthy postmenopausal women. Cancer Epidemiol Biomark. Prev. 2000; 9: 773-9.

33 Feskanich D, Sielaff B, Chong K, Buzzard I. Computerized collection and analysis of dietary intake information. Comput. Meth. Prog. Biol. 1989; 30: 47-57.

34 Martin HJ. A revised measure of approval motivation and its relationship to social desirability. J. Pers. Assess. 1984; 48: 508-16.

35 Marlowe D, Crowne D. Social desirability and responses to perceived situational demands. J. Consult. Clin. Psychol. 1961; 25: 109-15.
36 Bradlow HL, Sepkovic DW, Klug T, Osborne MP. Application of an improved ELISA assay to the analysis of urinary estrogen metabolites. Steroids 1998; 63(7-8): 406-13.

37 Hebert JR, Hurley T, Chiraboga DE, Barone JA. A comparison of selected nutrient intakes derived from three diet assessment methods used in a low-fat maintenance trial. Public Health Nutr. 1998; 1: 207-14

38 Buzzard IM, Faucett CL, Jeffrey RW, McBane L, McGovern $\mathrm{P}$, Baxter JS, et al. Monitoring dietary change in a low-fat diet intervention study: advantages of using 24-hour dietary recalls vs food records. J. Am. Diet. Assoc. 1996; 96: 574-9.

39 Fenwick GR, Heaney RK, Mullin WJ. Glucosinolates and their breakdown products in food and food plants. In: $C R C$ Critical Reviews in Food Science and Nutrition, 1983; 123200.

40 Steinmetz KA, Kushi LH, Bostick RM, Folsom AR, Potter JD. Vegetables, fruit, and colon cancer in the Iowa Women's Health Study. Am. J. Epidemiol. 1994; 139(1): 1-15.

41 McDanell R, McLean AEM, Hanley AB, Heaney RK, Fenwick GR. Differential induction of mixed-function oxidase activity in rat liver and intestine by diets containing processed cabbage: correlation with cabbage levels of glucosinolates and glucosinolate hydrolysis products. Food Chem. Tox. 1987; 25(5): 363-8.

42 Howard LA, Jeffrey EH, Wallig MA, Klein BP. Retention of phytochemicals in fresh and processed broccoli. J. Food Sci. 1997; 62(6): 1098-100.

43 Slominski BA, Campbell LD. Formation of indole glucosinolate breakdown products in autolyzed, streamed, and cooked Brassica vegetables. J. Agric. Food Chem. 1989; 37: 1297-302.

44 Bjeldanes LF, Kim J-Y, Grose KR, Bartholomew JC, Bradfield CA. Aromatic hydrocarbon responsiveness-receptor agonists generated from indole-3-carbinol in vitro and in vivo: comparisons with 2,3,7,8-tetrachlorodibenzo-p-dioxin. Proc. Natl. Acad. Sci. USA 1991; 88: 9543-7.

45 Kabat GC, Chang CJ, Sparano JA, Sepkovic DW, Hu X-P, Khalil A, et al. Urinary estrogen metabolites and breast cancer: a case-control study. Cancer Epidemiol. Biomark. Prev. 1997; 6: 505-9.

46 Zheng, W, Dunning, L, Jin, F, Holtzman, J. Correspondence re: GC Kabat et al., Urinary estrogen metabolites and breast cancer: a case-control study. Cancer Epidemiol. Biomark. Prev. 1998: 7: 85-6.

47 Bradlow HL, Telang NT, Sepkov DW, Osborne MP. 2Hydroxyestrone: the 'good' estrogen. J. Endocrinol. 1996; 150: S256-65.

48 Ursin G, London S, Stanczyk FZ, Gentzchein E, Paganini-Hill A, Ross RK, et al. Urinary 2-hydroxyestrone/16 $\alpha$-hydroxyestrone ratio and risk of breast cancer in postmenopausal women. J. Natl. Cancer Inst. 1999; 91(12): 1067-72.

49 Ho GH, Luo XW, Ji CY, Foo SC, Ng EH. Urinary 2/16hydroxyestrone ratio: correlation with serum insulin-like growth factor binding protein-3 and a potential biomarker of breast cancer. Ann. Acad. Med. Singapore 1998; 27: 294-9.

50 Meilahn E, DeStavola B, Allen D, Fentim I, Bradlow H, Sepkovic D, et al. Do urinary oestrogen metabolites predict breast cancer? Guernsey III cohort follow-up. Br. J. Cancer 1998; 78(9): 1250-5.

51 Walter-Sack I. Klotz U. Influence of diet and nutritional status on drug metabolism. Clin. Pharmacokinetics 1996; 31(1): $47-64$. 